\title{
Preventing Hypoglycemia Following Treatment of Hyperkalemia in Hospitalized Patients
}

\author{
Charlotte K Boughton, MBBS, PhD ${ }^{1,2}$; Danielle Dixon, MBBS 1,2; Emma Goble, MRPharmS 1,3; Alice Burridge, PhD',3; \\ Alison Cox, RN 1,2; Georgia Noble-Bell, RN, MSc ${ }^{1,2}$; Charlotte Bell, MRPharmS 1,3; Ben Fidler1,4; James Chudley 1,4; \\ Caroline Anderson, MRPharmS, FFCl',3; Gillian Cavell, MRPharmS, MSc ${ }^{1,3}$; Omar G Mustafa, MBChB, FRCP ${ }^{1,2 \star}$
}

\begin{abstract}
${ }^{1}$ King's Insulin Safety Group, King's College Hospital NHS Foundation Trust, London, United Kingdom; ${ }^{2}$ Department of Diabetes, King's College Hospital NHS Foundation Trust, London, United Kingdom; ${ }^{3}$ Department of Pharmacy and Medications safety, King's College Hospital NHS Foundation Trust, London, United Kingdom; ${ }^{4}$ Clinical Systems, King's College Hospital NHS Foundation Trust, London, United Kingdom.
\end{abstract}

Hypoglycemia is a serious complication following treatment of hyperkalemia with intravenous insulin. The aims of this study were to determine the incidence of hypoglycemia ( $\leq 3.9 \mathrm{mmol} / \mathrm{l}, 70 \mathrm{mg} / \mathrm{dL}$ ) and severe hypoglycemia $(<3.0$ $\mathrm{mmol} / \mathrm{l}, 54 \mathrm{mg} / \mathrm{dL}$ ) in noncritical care inpatients following treatment of hyperkalemia and to establish the risk factors predisposing to this complication. This was a single-center observational study reviewing the Electronic Patient Records of hyperkalemia treatment with intravenous insulin on the general wards of a large UK teaching hospital. A total of 662 episodes of hyperkalemia treated with insulin/dextrose were included. Among these episodes, 116 treatments (17.5\%) resulted in hypoglycemia and $47(7.1 \%)$ resulted in severe hypoglycemia. Lower pretreatment capillary blood glucose level, older age, and lower bodyweight were associated with a higher risk of posttreatment hypoglycemia. The incidence of hypoglycemia following hyperkalemia treatment in hospitalized patients is unacceptably high. Identifying individuals at high risk of hypoglycemia and adjusting prescriptions may reduce the incidence. Journal of Hospital Medicine 2019;14:284-287. Published online first February 20, 2019. (C) 2019 Society of Hospital Medicine

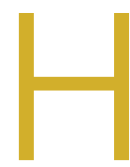

yperkalemia is common in hospitalized patients, with an estimated prevalence of $1 \%-10 \%$. ${ }^{1,2}$ Hyperkalemia can lead to life-threatening cardiac arrhythmias. The risk of arrhythmias increases with serum potassium values $>6.5 \mathrm{mmol} / \mathrm{L}$, and hyperkalemia is associated with increased in-hospital mortality. ${ }^{3}$ Treatment for hyperkalemia is indicated by a combination of the absolute serum potassium level, the rate of change of potassium level, and the presence of electrocardiogram abnormalities.

Intravenous insulin stimulates the sodium/potassium-ATP pump, leading to intracellular uptake of potassium. Recommendations vary regarding the optimal dosing of insulin and dextrose for the treatment of hyperkalemia. ${ }^{4}$

Hypoglycemia is a common complication following treatment of hyperkalemia with insulin/dextrose. The reported incidence in hospitalized patients ranges from $6 \%$ to $75 \%$ depending on the population studied, the doses of insulin/ dextrose administered, and the definition of hypoglycemia. ${ }^{5-8}$ Hypoglycemia itself is associated with increased morbidity and mortality in hospitalized patients. ${ }^{9}$

The aims of this study were to describe the incidence of hypoglycemia following hyperkalemia treatment with intra-

*Corresponding Author: Omar G Mustafa MBChB, FRCP; E-mail: omarmustafa@nhs.net; Telephone: (020) 3299-1588; Twitter: @OGMustafa

Received: October 11, 2018; Revised: December 16, 2018;

Accepted: December 16, 2018

(C) 2019 Society of Hospital Medicine DOI 10.12788/jhm.3145 venous insulin/dextrose in inpatients in a large (900-bed) UK teaching hospital and to determine the risk factors predisposing to hypoglycemia.

\section{METHODS}

We conducted a retrospective, single-center cohort study reviewing the Electronic Patient Records (EPR) of all adult (aged $\geq 18$ years) inpatients (excluding critical care) prescribed treatment for hyperkalemia with intravenous insulin from January 1, 2013, to March 1, 2017. Local hyperkalemia treatment guidelines included administration of 10 units of insulin and 100 $\mathrm{ml}$ of $20 \%$ glucose intravenously in accordance with national guidelines. ${ }^{10}$ The study received local approval.

Episodes occurring before May 1, 2015, were excluded because modification to the hyperkalemia prescription care bundle was implemented in April 2015 recommending standardized simultaneous insulin and dextrose administration and hourly capillary blood glucose (CBG) measurement for six hours following treatment. Episodes where no dextrose was prescribed or administered ( $n=435$ ) or where no CBG value was recorded within six hours after treatment were excluded ( $\mathrm{n}$ =63). All patients included in the analysis received the same insulin/dextrose treatment confirmed by electronic signature of the prescription.

Data extracted included patient demographics, laboratory values, and treatment and administration details. Pretreatment and posttreatment potassium measurements were taken within four hours before and after insulin/dextrose administration, respectively. Serum creatinine and estimated glomerular filtra- 
tion rate (eGFR) measurements were taken within six hours prior to treatment. Pretreatment CBG levels were measured within two hours of insulin/dextrose administration, and the lowest value within six hours after treatment was used for the analysis. We collected data on length of stay and mortality during oneyear follow-up.

Hypoglycemia and severe hypoglycemia were defined as CBG $\leq 3.9 \mathrm{mmol} / / \mathrm{l}(70 \mathrm{mg} / \mathrm{dL})$ and $<3.0 \mathrm{mmol} / \mathrm{l}(54 \mathrm{mg} / \mathrm{dL})$ in line with definitions used in the National Diabetes Inpatient Audit. ${ }^{11}$

Descriptive statistics are reported as mean $( \pm \mathrm{SD})$ or median (interquartile range [IQR]) values for continuous data or numbers and percentages for categorical data. All $P$ values are twotailed, and $P$ values $<.05$ were considered to indicate statistical significance. Chi-squared test and Student t test were used to assess differences for categorical and continuous variables between groups. The statistical analysis was performed using the SPSS software, version 25 (IBM).

\section{RESULTS}

A total of 662 episodes of hyperkalemia treatment with insulin/dextrose were included in the analysis. These episodes occurred over 445 admissions in 415 individuals. The median number of treatments/patient admission was 1.0 (range 1-11); 108 patients received more than one insulin/dextrose treatment during their admission. Mean pretreatment serum potassium level was $6.4 \pm 0.5 \mathrm{mmol} / \mathrm{l}$, and treatment reduced the serum potassium level by $0.6 \pm 0.6 \mathrm{mmol} / \mathrm{l}$.

\section{Patient Demographics}

Median age of the patients was 67 years (IOR 55.0-79.0), and $39.3 \%$ of episodes occurred in females (Table 1). Median weight of the patients was $76.6 \mathrm{~kg}$ (IQR 62.1-95.0). Diabetes was present in $31.1 \%$ of episodes. Renal impairment was common, with median creatinine levels being $166 \mu \mathrm{mol} / \mathrm{I}$ (IOR 113-256) and $1.9 \mathrm{mg} / \mathrm{dL}$ (IQR 1.3-2.9) and eGFR being $29.0 \mathrm{ml} /$ $\mathrm{min} / 1.73 \mathrm{~m}^{2}$ (IQR 19.0-45.0), and $11 \%$ of episodes occurred in patients requiring acute or chronic dialysis. Median length of stay was 19.5 days (IOR 9.8-49.1). Inpatient mortality was 13\%, and one-year mortality was $19.4 \%$.

\section{Incidence}

Hypoglycemia occurred following 116 of 662 hyperkalemia treatments administered (17.5\%), and severe hypoglycemia occurred after 47 of 662 treatments (7.1\%).

\section{Risk Factors}

The median age of patients with hypoglycemia was significantly greater than that of patients without hypoglycemia (71.0 years [54.8-83.5] vs 67.0 years [55.0-77.0]; $P=.023$ ) (Table 2). There were no significant differences in gender, degree of renal impairment, or requirement for renal replacement therapy between the groups with and without hypoglycemia.

Hypoglycemia occurred in patients who were, on average, 15 $\mathrm{kg}$ lighter than those who did not have hypoglycemia (median body weight $66.1 \mathrm{~kg}$ [55.4-72.5] vs $81.0 \mathrm{~kg}$ [63.1-96.0]; $P<.001)$.
TABLE 1. Patient Demographics of Those Treated for Hyperkalemia. Data are Presented as Median and IOR

\begin{tabular}{ll}
\hline Age on admission (years) & Total $(\mathbf{n}=662)$ \\
\hline Gender ( $\mathrm{n}, \%$ Female) & $67.0(55.0-79.0)$ \\
\hline Weight $(\mathrm{kg})$ & $260(39.3)$ \\
\hline Diabetes, $\mathrm{n}(\%)$ & $76.6(62.1-95.0)$ \\
\hline Creatinine ( $\mu$ mol/l, mg/dL) & $206(31.1)$ \\
\hline eGFR (ml/minute/1.73 $\left.\mathrm{m}^{2}\right)$ & $166(113-256), 1.88(1.28-2.90)$ \\
\hline Renal Replacement Therapy, $\mathrm{n}(\%)$ & $29.0(19.0-45.0)$ \\
\hline Length of Stay (days) & $73(11.0)$ \\
\hline Inpatient Mortality, $\mathrm{n}(\%)^{*}$ & $19.5(9.8-49.1)$ \\
\hline Mortality within 1 year, $\mathrm{n}(\%)^{*}$ & $79(13.0)$ \\
\hline
\end{tabular}

Abbreviations: eGFR, estimated glomerular filtration rate; IQR, interquartile range.

Pretreatment CBG was lower in those who had hypoglycemia following treatment, the levels being $5.8 \mathrm{mmol} / \mathrm{l}$ (5.0-7.3), $104 \mathrm{mg} / \mathrm{dL}$ (90-131) vs $8.7 \mathrm{mmol} / \mathrm{l}$ (6.4-11.4), $157 \mathrm{mg} / \mathrm{dL}$ (115205; $P<.001)$.

There was a nonsignificant trend toward an increased prevalence of diabetes in patients without hypoglycemia (32.6\% vs $24.1 \% ; P=.074)$.

\section{DISCUSSION}

This study reports the incidence of iatrogenic hypoglycemia following intravenous insulin treatment for hyperkalemia in a large cohort of general medical and surgical inpatients and describes the risk factors predisposing to this important complication.

The incidence rates of hypoglycemia and severe hypoglycemia in our study were $17.5 \%$ and $7.1 \%$, respectively. These rates are greater than previously observed in a smaller US study undertaken in a similar population, which reported an incidence of $8.7 \%$ of hypoglycemia and $2.3 \%$ of severe hypoglycemia, although it included five different insulin/dextrose prescriptions. ${ }^{8}$ A similar incidence of hypoglycemia (17\%) was reported in patients treated for hyperkalemia in an Emergency Department in the United States. ${ }^{12}$

Variables that increased the risk of hypoglycemia in the present study included older age, lower body weight, and lower pretreatment CBG level. These risk factors have been reported in previous studies, although inconsistently. Pretreatment CBG is an important predictor of hypoglycemia following treatment for hyperkalemia and has been observed in patients in the emergency department ${ }^{12}$ and in patients with renal impairment. ${ }^{6,7}$ In the present study, lower body weight was observed in patients with hypoglycemia compared with those without hypoglycemia. Weight-based insulin dosing (0.1 units $/ \mathrm{kg}$ ) for hyperkalemia has been associated with less hypoglycemia 
TABLE 2. Risk Factors for Hypoglycemia

\begin{tabular}{|c|c|c|c|}
\hline & $\begin{array}{c}\text { No Hypoglycemia } \\
(n=546)\end{array}$ & $\begin{array}{c}\text { Hypoglycemia } \\
\leq 3.9 \mathrm{mmol} / \mathrm{l}(\mathrm{n}=116)\end{array}$ & $P$ \\
\hline Age on admission (years) & $67.0(55.0-77.0)$ & $71.0(54.8-83.5)$ & .023 \\
\hline Female gender, $n(\%)$ & $215(39.4)$ & $45(38.8)$ & .907 \\
\hline Weight (kg) & $81.0(63.1-96.0)$ & $66.1(55.4-72.5)$ & $<.001$ \\
\hline Creatinine $(\mu \mathrm{mol} / \mathrm{l})$ & $168(113-255)$ & $156(112-271)$ & .727 \\
\hline eGFR (ml/minute/1.73 m2) & $29.5(19.0-46.0)$ & $29.0(17.0-41.0)$ & .722 \\
\hline Renal Replacement Therapy, $n$ (\%) & $58(10.6)$ & $15(12.9)$ & .472 \\
\hline Insulin in previous 24 hours, $n$ (\%) & $348(63.7)$ & $57(49.1)$ & .003 \\
\hline
\end{tabular}

compared with fixed insulin doses (10 units) without affecting the potassium-lowering effect. ${ }^{13}$

The degree of renal impairment did not affect the risk of hypoglycemia. Chronic kidney disease is associated with increased insulin resistance, which may attenuate the hypoglycemic response to insulin. ${ }^{14}$ Patients with renal impairment may experience delayed hypoglycemia, which may not have been captured although the posttreatment blood glucose values extended to six hours.

The increased risk of hypoglycemia in older patients treated for hyperkalemia is of concern given the lack of counterregulatory response and reduced symptoms of hypoglycemia in older adults. This association has not been reported in other studies, ${ }^{8,12}$ however, the average age of subjects in our cohort was higher than that in other studies (67 vs 57 years). Although age did not correlate with weight, older adults may have reduced carbohydrate intake and mild renal impairment affecting insulin clearance.

Hospitalized patients treated for hyperkalemia in the present study had a greater inpatient mortality rate (13\%) than the general inpatient population at the same hospital (3\%). ${ }^{15} \mathrm{Hy}-$ perkalemia often occurs in individuals with comorbidities and is associated with an increased risk of all-cause mortality ${ }^{3}$

Mean pretreatment potassium level was $6.4 \mathrm{mmol} / \mathrm{l}$ in this study. Evidence-based criteria for treatment thresholds are lacking. Acute potassium increases are associated with cardiac mortality, whereas elevated potassium levels in patients with chronic kidney disease taking renin-angiotensin system drugs are often not treated as emergencies unless significant electrocardiogram (ECG) changes are apparent. It is likely that some hyperkalemia treatments are unnecessary, which is important given the high risk of treatment-related complications.

To our knowledge, this is the largest analysis of hypoglycemic episodes following treatment of hyperkalemia in medical and surgical inpatients. This is a single-center study, thereby limiting its generalizability; however, the patient characteristics are likely similar to those reported from other large, urban institutions.

Treatment prescriptions in our study were consistent due to the electronic prescribing care bundle, allowing us to compare the risk factors for hypoglycemia with standardized prescriptions. Point-of-care CBG measurements can be less accurate at low blood glucose levels; however, laboratory glucose levels are obtained much less frequently, underestimating incidence, due to the need for prompt hypoglycemia treatment without delaying for laboratory glucose measurement.

The dataset was not complete and depended on healthcare professionals entering some data. We did not assess the clinical consequences of hypoglycemia.

\section{FUTURE WORK}

An increasing proportion of hospitals are utilizing Electronic Prescribing Systems with the potential to improve patient safety by standardizing prescriptions. However, despite standardized prescriptions, $37 \%$ of prescriptions for concurrent dextrose were not administered with insulin and $8.6 \%$ of patients had no CBG monitoring within six hours after insulin administration.

We propose to integrate the risk factors identified in this study into a decision support tool embedded within electronic prescriptions and medication administration. This will auto-populate with data from the EPR and identify individuals at high risk of hypoglycemia following hyperkalemia treatment. The decision support tool will then advise a prescription with a higher volume of dextrose and/or lower insulin dose to mitigate this risk.

\section{CONCLUSION}

Hyperkalemia treatment with insulin was associated with high incidence of hypoglycemia. Decision support tools highlight- 
ing individuals at high risk of hypoglycemia may reduce this incidence.

Disclosures: The authors have nothing to disclose.

\section{References}

1. Stevens MS, Dunlay RW. Hyperkalemia in hospitalized patients. Int Urol Nephrol. 2000;32(2):177-180. doi: 10.1023/A:1007135517950.

2. Khanagavi J, Gupta T, Aronow WS, et al. Hyperkalemia among hospitalized patients and association between duration of hyperkalemia and outcomes. Arch Med Sci. 2014;10(2):251-257. doi: 10.5114/aoms.2014.42577.

3. Einhorn LM, Zhan M, Hsu VD, et al. The frequency of hyperkalemia and its significance in chronic kidney disease. Arch Intern Med. 2009;169(12):11561162. doi: 10.1001/archinternmed.2009.132.

4. Harel Z, Kamel KS. Optimal dose and method of administration of intravenous insulin in the management of emergency hyperkalemia: a systematic review. PLoS One. 2016;11(5):e0154963. doi: 10.1371/journal.pone.0154963.

5. Allon M, Copkney C. Albuterol and insulin for treatment of hyperkalemia in hemodialysis patients. Kidney Int. 1990;38(5):869-872. doi: 10.1038/ki.1990.284.

6. Apel J, Reutrakul S, Baldwin D. Hypoglycemia in the treatment of hyperkalemia with insulin in patients with end-stage renal disease. Clin Kidney J. 2014;7(3):248-250. doi: 10.1093/ckj/sfu026.

7. Coca A, Valencia AL, Bustamante J, Mendiluce A, Floege J. Hypoglycemia following intravenous insulin plus glucose for hyperkalemia in patients with impaired renal function. PLoS One. 2017;12(2):e0172961. doi: 10.1371/journal.pone.0172961.
8. Schafers S, Naunheim R, Vijayan A, Tobin G. Incidence of hypoglycemia following insulin-based acute stabilization of hyperkalemia treatment. J Hosp Med. 2012;7(3):239-242. doi: 10.1002/jhm.977.

9. Nirantharakumar K, Marshall T, Kennedy A, Narendran P, Hemming K, Coleman JJ. Hypoglycaemia is associated with increased length of stay and mortality in people with diabetes who are hospitalized. Diabet Med. 2012;29(12):e445-e448. doi: 10.1111/dme.12002.

10. UK Renal Association Clinical Practice Guidelines: Treatment of acute hyperkalaemia in adults. https://renal.org/guidelines/. Published March 2014. Accessed 1 October 2018.

11. National Diabetes Inpatient Audit, England and Wales, 2017 - Full Report. NHS Digital. https://digital.nhs.uk/data-and-information/publications/ statistical/national-diabetes-inpatient-audit/national-diabetes-inpatient-audit-nadia-2017. Published 14 March 2018. Accessed 5 November 2018.

12. Scott NL, Klein L, Cales E, Driver B. Hypoglycemia as a complication of intravenous insulin to treat hyperkalemia in the emergency department. Am J Emerg Med. 2018; 30379-30386. doi: 10.1016/j.ajem.2018.05.016.

13. Wheeler DT, Schafers SJ, Horwedel TA, Deal EN, Tobin GS. Weight-based insulin dosing for acute hyperkalemia results in less hypoglycemia. J Hosp Med. 2016;11(5):355-357. doi: 10.1002/jhm.2545.

14. Spoto B, Pisano A, Zoccali C. Insulin resistance in chronic kidney disease: a systematic review. Am J Physiol Renal Physiol. 2016;311(6):F1087-F1108. doi: 10.1152/ajprenal.00340.2016.

15. Summary Hospital-level Mortality Indicator (SHMI) - Deaths associated with hospitalisation, England, April 2017 - March 2018. NHS Digital. https:// digital.nhs.uk/data-and-information/publications/clinical-indicators/shmi/ archive/shmi-april-2017---march-2018. Published 20 September 2018. Accessed 5 November 2018. 